\title{
Wireless Network Coding with Intelligent Reflecting Surfaces
}

\author{
Amanat Kafizov, Student Member, IEEE, Ahmed Elzanaty, Member, IEEE, Lav R. Varshney, Senior \\ Member, IEEE, Mohamed-Slim Alouini, Fellow, IEEE
}

\begin{abstract}
Conventional wireless techniques are becoming inadequate for beyond-5G networks due to latency and bandwidth considerations. To improve the error performance of wireless communication systems, we propose physical layer network coding (PNC) in an intelligent reflecting surface (IRS)-assisted environment. We consider an IRS-aided butterfly network, where we propose an algorithm for obtaining the optimal IRS phases. Analytic expressions for the bit error rate (BER) are derived. Numerical results demonstrate that the proposed scheme significantly improves the BER performance. For instance, the BER at the relay in the presence of a 32-element IRS is three orders of magnitudes less than that without an IRS.
\end{abstract}

Index Terms-Intelligent reflecting surfaces; network coding; butterfly network; performance analysis

\section{INTRODUCTION}

Conventional wireless communication techniques are becoming inadequate for beyond fifth-generation (5G) networks because they, in general, have excessive network latency and low spectral efficiency [1]. Network coding is a promising solution to increase the throughput of wireless networks [2], [3], which relies on the ability of the relay (router) to perform more complex operations than just forwarding [4]. In physical layer network coding (PNC) specifically, the physical broadcast nature of wireless links, which generally causes deleterious interference, is exploited to increase the network throughput [2]-[6]. However, PNC with higher data rates suffers from high path loss and is susceptible to blockages. These limitations suggest future sustainable networks, where the propagation channel itself can be controlled.

In this regard, so-called intelligent reflecting surfaces (IRSs) have been demonstrated to overcome some of the issues associated with PNC. An IRS is a flat surface which comprises many small passive elements, each of which can independently introduce phase changes to the incident signals [7]. Thus, unlike traditional relays, it does not need a dedicated energy source or consume any transmit power. An IRS can be easily integrated into walls, ceilings, and building facades [8][11]. IRSs can improve the bit error rate (BER) performance of over-air-computation (OAC) techniques, which integrate wireless communication and computation [12]-[15]. This is achieved by smartly tuning the IRS phase profile, thereby significantly boosting the received signal power. In a sense,

A. Kafizov, A. Elzanaty, and M.-S. Alouini are with Computer, Electrical, and Mathematical Science and Engineering (CEMSE) Division, King Abdullah University of Science and Technology (KAUST), Thuwal, Saudi Arabia (e-mail: \{amanat.kafizov, ahmed.elzanaty, slim.alouini\}@kaust.edu.sa).

L. R. Varshney is with the Coordinated Science Laboratory, University of Illinois Urbana-Champaign, Urbana, IL, USA (e-mail: varshney@illinois.edu).

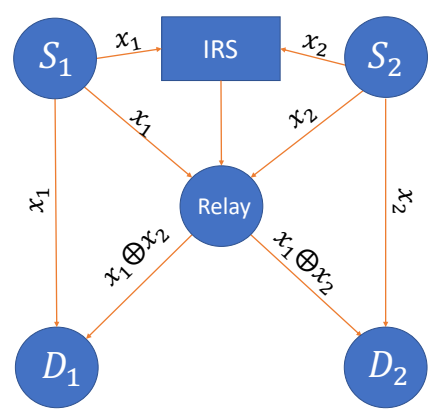

Fig. 1. IRS-aided butterfly network.

PNC is a kind of OAC as it is based on the linear or nonlinear aggregation of signals by the wireless medium; yet it has remained unstudied in the presence of IRSs.

In this paper, we propose a PNC scheme in an IRS-assisted environment to enhance the error performance of wireless communication systems, especially in unfavorable channel conditions. As a proof of concept, we focus on the butterfly network, usually adopted as the basic setting for network coding [6], [16]. In this setting, the relay computes networkcoded packets directly from the received signal. To improve BER performance, we optimize the IRS profile. Although, the problem is non-convex, we propose an efficient optimization algorithm based on matrix lifting. Then, we derive the analytic BER of an IRS-aided butterfly network to judge error performance. Numerical results verify the significant improvement of BER performance due to the proposed scheme.

For notation, the probability of an event and expectation of a random variable are denoted by $\mathbb{P}\{\cdot\}$ and $\mathbb{E}[\cdot]$, respectively. Capital bold letters, e.g., $\mathbf{X}$, and small bold letters, e.g., $\mathbf{x}$, denote matrices and vectors, respectively. The notation $\operatorname{diag}(\mathbf{x})$ represents a diagonal matrix with diagonal $\mathbf{x}$, whereas $\operatorname{tr}(\cdot)$ denotes the matrix trace. The transpose and Hermitian of vectors or matrices are denoted as $(\cdot)^{T}$ and $(\cdot)^{H}$. The $l$ th row and column of a matrix $\mathbf{X}$ are denoted by $\mathbf{x}_{(l)}$ and $\mathbf{x}_{l}$, respectively. The $(k, l)$ th element of matrix $\mathbf{X}$ is denoted as $x_{k l}$. The real part of complex number $z$ is denoted by $\Re(z)$. The $\ell_{2}$-norm of a vector $\mathbf{x}$ is denoted by $\|\mathbf{x}\|$.

\section{SYSTEM MODEL}

This paper considers the IRS-aided butterfly network shown in Fig. 1, where sources $S_{1}$ and $S_{2}$ want to deliver their messages to destinations $\mathrm{D}_{1}$ and $\mathrm{D}_{2}$. For simplicity, we assume each source and destination node has a single antenna to transmit and receive, respectively, whereas the relay node, 
operating in half-duplex mode, is equipped with $N_{\mathrm{r}}=2$ antennas to receive and transmit. To enhance communication performance from $N_{\mathrm{s}}=2$ sources to relay, we propose deploying an IRS with $M$ reflecting elements, as in Fig. 1. We assume that the destination nodes are quite far from IRS such that the received power from the IRS at the destination is negligible compared to the direct link from the source node. Hence, links from the IRS to $D_{1}$ and $D_{2}$ are ignored.

Let $P$ and $s_{i}$ be the transmit power and the transmitted symbol of source $i$, respectively. Hence, the signal transmitted by source $i$ is $x_{i}=\alpha_{i} s_{i}$, where $\alpha_{i}=\sqrt{P / \sigma_{s, i}^{2}}$. The power of $s_{i}$ is $\mathbb{E}\left[\left|s_{i}\right|^{2}\right]=\sigma_{s, i}^{2}$. We assume binary phase shift keying (BPSK) modulation at source nodes, but this can be extended to quadrature phase shift keying (QPSK). The noise at the $j$ th antenna of the relay is assumed to be complex Gaussian with zero mean and $\sigma^{2}$ variance, i.e., $n_{j} \sim \mathcal{C N}\left(0, \sigma^{2}\right)$.

There are two stages in PNC. In the first stage, source nodes simultaneously broadcast their data to the relay and to destination nodes. We assume that the signals from $\mathrm{S}_{1}$ and $\mathrm{S}_{2}$ arrive at the relay with symbol-level synchronization both via a direct channel and a reflecting channel (via IRS) [3]. Let $\mathbf{H}^{\mathbf{u r}} \in \mathbb{C}^{N_{\mathrm{r}} \times N_{\mathrm{s}}}, \mathbf{H}^{\mathbf{u i}} \in \mathbb{C}^{M \times N_{\mathrm{s}}}, \mathbf{H}^{\mathbf{i r}} \in \mathbb{C}^{N_{\mathrm{r}} \times M}, h_{\mathrm{D}_{1}}^{\mathrm{S}_{1}} \in \mathbb{C}^{1 \times 1}$ and $\mathbf{h}_{\mathrm{D}_{1}}^{r} \in \mathbb{C}^{1 \times N_{\mathrm{r}}}$ be equivalent channels from sources directly to relay, from sources to IRS, from IRS to relay, from $S_{1}$ to $D_{1}$, and from relay to $D_{1}$, respectively. Hence, the received signal at the relay can be written as

$$
\mathbf{r}=\left(\mathbf{H}^{\mathrm{ir}} \boldsymbol{\Theta} \mathbf{H}^{\mathrm{ui}}+\mathbf{H}^{\mathrm{ur}}\right) \mathbf{x}+\mathbf{n} \triangleq \mathbf{H x}+\mathbf{n},
$$

where $\boldsymbol{\Theta}=\operatorname{diag}(\mathbf{v})$ is the IRS diagonal phase shift matrix with $\mathbf{v}=\left[e^{j \theta_{1}}, \ldots, e^{j \theta_{M}}\right]_{T}^{T}$ and $\theta_{m} \in[0,2 \pi], \mathbf{x}=\left[x_{1}, x_{2}\right]^{T}$ and $\mathbf{n}=\left[n_{1}, n_{2}, \ldots, n_{N_{\mathrm{r}}}\right]^{T}$ are the signal and noise vectors, respectively. Simultaneously, the signal received at $\mathrm{D}_{1}$ directly from $S_{1}$ is

$$
r_{\mathrm{D}_{1}}^{\mathrm{S}_{1}} \triangleq h_{\mathrm{D}_{1}}^{\mathrm{S}_{1}} x_{1}+n_{\mathrm{D}_{1}}^{\mathrm{S}_{1}},
$$

where $n_{\mathrm{D}_{1}}^{\mathrm{S}_{1}} \sim \mathcal{C N}\left(0, \sigma^{2}\right)$. After channel inversion, $y_{\mathrm{D}_{1}}^{\mathrm{S}_{1}} \triangleq x_{1}+$ $\widetilde{n}_{\mathrm{D}_{1}}^{\mathrm{S}_{1}}$, where $\widetilde{n}_{\mathrm{D}_{1}}^{\mathrm{S}_{1}} \sim \mathcal{C N}\left(0, \sigma_{\mathrm{S}_{1}, \mathrm{D}_{1}}^{2}\right)$ and $\sigma_{\mathrm{S}_{1}, \mathrm{D}_{1}}^{2}=\sigma^{2} /\left|h_{\mathrm{D}_{1}}^{\mathrm{S}_{1}}\right|^{2}$.

In the second stage, the relay computes an exclusive $\mathrm{OR}$ logical operator (XOR) of the signals from $\mathrm{S}_{1}$ and $\mathrm{S}_{2}$, i.e., $x_{\oplus} \triangleq x_{1} \oplus x_{2}$. Here, $x_{\oplus}$ is referred to as the network-coded signal. Then, the relay broadcasts the network-coded signal to $\mathrm{D}_{1}$ and $\mathrm{D}_{2}$. The nodes $\mathrm{D}_{1} / \mathrm{D}_{2}$ can compute $x_{2} / x_{1}$ by XORing $x_{1} / x_{2}$ and a network-coded signal from the relay.

In naive network-layer network coding (NNC), the relay first decodes $x_{1}$ and $x_{2}$ individually, and then computes the network-coded signal, $x_{\oplus}$. However, such schemes are suboptimal in terms of the desired output since they do not consider the fact that only $x_{\oplus}$ is needed at the relay rather than individual signals $x_{1}$ and $x_{2}$ [3], [6].

Therefore, we propose a PNC approach where the relay computes $x_{\oplus}$ without decoding $x_{1}$ and $x_{2}$ individually. In fact, it is more useful to get $x_{\oplus}$ from $x_{1}+x_{2}$ and $x_{1}-x_{2}$, which can be calculated directly from $\mathbf{r}$ via matrix multiplication. Hence, the relay can get $x_{\oplus}$ almost at full rate [6], [17]. As such, the received signal in (1) can be rewritten as

$$
\mathbf{r}=\left(\mathbf{H D}^{-1}\right)(\mathbf{D} \mathbf{x})+\mathbf{n}=\widetilde{\mathbf{H}} \widetilde{\mathbf{x}}+\mathbf{n},
$$

where

$$
\mathbf{D}=2 \mathbf{D}^{-1}=\left[\begin{array}{cc}
1 & 1 \\
1 & -1
\end{array}\right]
$$

is the sum-difference matrix, and $\widetilde{\mathbf{x}}$ is

$$
\widetilde{\mathbf{x}}=\left[\begin{array}{l}
\widetilde{x}_{1} \\
\widetilde{x}_{2}
\end{array}\right] \triangleq \mathbf{D} \mathbf{x}=\left[\begin{array}{l}
x_{1}+x_{2} \\
x_{1}-x_{2}
\end{array}\right] .
$$

The vector $\widetilde{\mathbf{x}}$ is estimated from $\mathbf{r}$ using a linear operator as

$$
\mathbf{y}=\mathbf{G r},
$$

where $\mathbf{G} \in \mathbb{C}^{N_{\mathrm{s}} \times N_{\mathrm{r}}}$ is the beamforming matrix at the relay, to be designed in Section IV. We consider that the relay can estimate the channel state information (CSI) perfectly.

Let us define the estimation of $x_{1}$ and $x_{2}$ at $\mathrm{D}_{1}$ as $\widehat{x}_{1}$ and $\widehat{x}_{2}$, respectively. The estimation of $x_{\oplus}$ at the relay is defined as $\widehat{x}_{\oplus, \mathrm{r}}$ whereas the estimation of $\widehat{x}_{\oplus, \mathrm{r}}$ at $\mathrm{D}_{1}$ is defined as $\widehat{x}_{\oplus, \mathrm{D}_{1}}$. The likelihood estimators designed in Section V-A are considered to estimate $x_{1}$ from $y_{\mathrm{D}_{1}}^{\mathrm{S}_{1}}, x_{\oplus}$ from $\mathbf{y}$, and $\widehat{x}_{\oplus, \mathrm{r}}$ from $y_{\mathrm{D}_{1}}^{r}$, where $y_{\mathrm{D}_{1}}^{r}$ is the received signal at $\mathrm{D}_{1}$ from the relay after the channel inversion with variance $\sigma_{r, \mathrm{D}_{1}}^{2}=\sigma^{2} /\left\|\mathbf{h}_{\mathrm{D}_{1}}^{r}\right\|^{2}$.

\section{Problem Formulation}

In this section, we aim to design the optimal linear operator, i.e., the beamforming matrix $\mathbf{G}$, and the IRS phase profile $\Theta$ to minimize the BER at the destinations. For instance, without loss of generality, we consider the BER at $\mathrm{D}_{1}$, i.e., $\mathbb{P}_{\mathrm{D}_{1}} \triangleq$ $\mathbb{P}\left(\widehat{x}_{2} \neq x_{2}\right)$, which can be derived as

$$
\begin{aligned}
\mathbb{P}_{\mathrm{D}_{1}} & =\left[1-\mathbb{P}_{\mathrm{D}_{1}}^{\mathrm{S}_{1}}\right] \mathbb{P}_{\mathrm{D}_{1}}^{\mathrm{r}}\left[1-\mathbb{P}_{\oplus}^{\mathrm{r}}(\boldsymbol{\Theta}, \mathbf{G})\right]+\mathbb{P}_{\mathrm{D}_{1}}^{\mathrm{S}_{1}} \mathbb{P}_{\mathrm{D}_{1}}^{\mathrm{r}} \mathbb{P}_{\oplus}^{\mathrm{r}}(\boldsymbol{\Theta}, \mathbf{G})+\mathbb{P}_{\mathrm{D}_{1}}^{\mathrm{S}_{1}} \\
& \times\left[1-\mathbb{P}_{\oplus}^{\mathrm{r}}(\boldsymbol{\Theta}, \mathbf{G})\right]\left[1-\mathbb{P}_{\mathrm{D}_{1}}^{\mathrm{r}}\right]+\left[1-\mathbb{P}_{\mathrm{D}_{1}}^{\mathrm{S}_{1}}\right]\left[1-\mathbb{P}_{\mathrm{D}_{1}}^{\mathrm{r}}\right] \mathbb{P}_{\oplus}^{\mathrm{r}}(\boldsymbol{\Theta}, \mathbf{G}),(7)
\end{aligned}
$$

where $\mathbb{P}_{\mathrm{D}_{1}}^{\mathrm{S}_{1}} \triangleq \mathbb{P}\left(\widehat{x}_{1} \neq x_{1}\right), \mathbb{P}_{\oplus}^{\mathrm{r}}(\mathbf{\Theta}, \mathbf{G}) \triangleq \mathbb{P}\left(\widehat{x}_{\oplus, \mathrm{r}} \neq x_{\oplus}\right)$, and $\mathbb{P}_{\mathrm{D}_{1}}^{\mathrm{r}} \triangleq \mathbb{P}\left(\widehat{x}_{\oplus, \mathrm{D}_{1}} \neq \widehat{x}_{\oplus, \mathrm{r}}\right)$ are the BERs for the estimated $x_{1}$ at $\mathrm{D}_{1}$, estimated $x_{\oplus}$ at the relay, and estimated $\widehat{x}_{\oplus, \mathrm{r}}$ at $\mathrm{D}_{1}$, respectively. Note that the event $\left(\widehat{x}_{2} \neq x_{2}\right)$ at $\mathrm{D}_{1}$ occurs when the number of links with error is odd; therefore, $\mathbb{P}_{\mathrm{D}_{1}}$ is derived as (7). For example, $\mathbb{P}_{\mathrm{D}_{1}}^{\mathrm{S}_{1}}\left[1-\mathbb{P}_{\oplus}^{\mathrm{r}}(\boldsymbol{\Theta}, \mathbf{G})\right]\left[1-\mathbb{P}_{\mathrm{D}_{1}}^{\mathrm{r}}\right]$ in (7) considers that $\widehat{x}_{1} \neq x_{1}$ while $\widehat{x}_{\oplus, \mathrm{r}}=x_{\oplus}$ and $\widehat{x}_{\oplus, \mathrm{D}_{1}}=\widehat{x}_{\oplus, \mathrm{r}}$. The analytical expressions for BERs $\mathbb{P}_{\mathrm{D}_{1}}^{\mathrm{S}_{1}}, \mathbb{P}_{\oplus}^{\mathrm{r}}$, and $\mathbb{P}_{\mathrm{D}_{1}}^{\mathrm{r}}$ are derived in Section V-B. Since there is no link from IRS to $\mathrm{D}_{1}$, only $\mathbb{P}_{\oplus}^{\mathrm{r}}(\boldsymbol{\Theta}, \mathbf{G})$ depends on $\mathbf{G}$ and $\boldsymbol{\Theta}$ in (7). Hence, $\mathbb{P}_{\mathrm{D}_{1}}$ can be minimized by minimizing $\mathbb{P}_{\oplus}^{r}(\boldsymbol{\Theta}, \mathbf{G})$ with respect to (w.r.t.) $\mathbf{G}$ and $\boldsymbol{\Theta}$. To improve $\mathbb{P}_{\oplus}^{\mathrm{r}}(\boldsymbol{\Theta}, \mathbf{G})$, we can optimize mean-squared error (MSE) for the recovery of $\widetilde{\mathbf{x}}$ from $\mathbf{r}$ w.r.t. $\mathbf{G}$ and $\Theta$. In this regard, the optimization problem can be formulated as

$$
\begin{array}{ll}
\underset{\boldsymbol{\Theta}, \mathbf{G}}{\operatorname{minimize}} & \mathrm{MSE} \triangleq \mathbb{E}\left[\|\mathbf{y}(\boldsymbol{\Theta}, \mathbf{G})-\widetilde{\mathbf{x}}\|^{2}\right] \\
\text { subject to } & 0 \leq \theta_{m} \leq 2 \pi, m \in\{1,2, \ldots, M\} .
\end{array}
$$

The MSE between the estimated value $\mathbf{y}$ and target parameter $\widetilde{\mathbf{x}}$ can be computed as

$$
\begin{aligned}
\mathrm{MSE} & =\operatorname{tr}\left(\mathbb{E}\left\{(\mathbf{G r}-\widetilde{\mathbf{x}})\left(\mathbf{r}^{H} \mathbf{G}^{H}-\widetilde{\mathbf{x}}^{H}\right)\right\}\right) \\
& =\operatorname{tr}\left(\mathbf{G}\left(P \mathbf{H} \mathbf{H}^{H}+\sigma^{2} \mathbf{I}\right) \mathbf{G}^{H}-2 \Re\left\{\mathbf{G} P \mathbf{H D}^{H}\right\}+2 P \mathbf{I}\right) .
\end{aligned}
$$

The joint optimization over the beamforming matrix and IRS profile in (8) is challenging. In Section IV, we provide an efficient algorithm for the optimization problem. 


\section{Optimization AlgORITHM}

We propose an algorithm to optimize IRS phases while fixing the beamforming matrix in (8). In particular, for a fixed $\Theta$ in (8), the optimization problem is a quadratic unconstrained problem in $\mathbf{G}$ with closed-form solution, i.e.,

$$
\mathbf{G}=P \mathbf{D} \mathbf{H}^{H}\left(P \mathbf{H} \mathbf{H}^{H}+\sigma^{2} \mathbf{I}\right)^{-1},
$$

which is the minimum mean square error (MMSE) estimator for $\mathbf{G}$. To optimize the phases of IRS for fixed $\mathbf{G}$, we propose the matrix lifting technique [18]. First, the objective function should be written in terms of $\mathbf{v}$, i.e., MSE can be written as

$$
\begin{aligned}
& \mathrm{MSE} \triangleq P \sum_{i^{\prime}=1}^{N_{\mathrm{s}}} \sum_{j=1}^{N_{\mathrm{r}}}\left(\sum_{j=1}^{N_{\mathrm{r}}} \mathbf{g}_{j}^{H} \mathbf{g}_{j^{\prime}}\left(b^{j i^{\prime}}\right)^{H}\right) b^{j^{\prime} i^{\prime}}+2 P N_{\mathrm{s}} \\
& -2 P \Re\left(\sum_{i=1}^{N_{\mathrm{s}}} \sum_{j=1}^{N_{\mathrm{r}}} b^{j i}\left(g_{1 j}+(-1)^{i+1} g_{2 j}\right)\right)+\sum_{i=1}^{N_{\mathrm{s}}} \sigma_{i}{ }^{2},
\end{aligned}
$$

where $b^{j i}=\phi^{j i} \mathbf{v}+h_{j i}^{u r}$ and $\phi^{j i}=\mathbf{h}_{(j)}^{\mathrm{ir}} \operatorname{diag}\left(\mathbf{h}_{i}^{\text {ui }}\right)$. By substituting (10) in (11), we can write the optimization problem (8) for a fixed $\mathbf{G}$ as

$$
\begin{array}{ll}
\underset{\mathbf{v}}{\operatorname{minimize}} & \mathrm{MSE} \\
\text { subject to } & |\mathbf{v}(m)|^{2}=1, m \in\{1,2, \ldots, M\} .
\end{array}
$$

The unit modulus constraint, (12b), induces non-convexity. Thus, we apply a matrix lifting technique, which reformulates (12) as a rank-one optimization problem using matrix

$$
\mathbf{V}=\left[\begin{array}{l}
\mathbf{v} \\
1
\end{array}\right]\left[\begin{array}{ll}
\mathbf{v}^{H} & 1
\end{array}\right]=\left[\begin{array}{cc}
\mathbf{v v}^{H} & \mathbf{v} \\
\mathbf{v}^{H} & 1
\end{array}\right]
$$

followed by difference-of-convex functions (DC) representation of the rank-one constraint [18]. For $\mathbf{V}$, we have the following constraints: $\operatorname{rank}(\mathbf{V}) \leq 1, \mathbf{V} \succeq 0$, and $\mathbf{V}(k, k)=1$, $\forall k \in\{1, \ldots, M+1\}$. Let us rewrite (11) in terms of $\mathbf{V}$ and remove all the terms that do not depend on $\mathbf{v}$ as

$$
\begin{aligned}
& \mathrm{MSE} \propto \sum_{i^{\prime}=1}^{N_{\mathrm{s}}} \sum_{j^{\prime}=1}^{N_{\mathrm{r}}} \sum_{j=1}^{N_{\mathrm{r}}} \operatorname{tr}\left(\mathbf{g}_{j}^{H} \mathbf{g}_{j^{\prime}}\left[\begin{array}{cc}
\phi^{j i^{\prime H} H} \phi^{j^{\prime} i^{\prime}} & \phi^{j i^{\prime H}} h_{j^{\prime} i^{\prime}}^{u r} \\
h_{j i^{\prime}}^{* u r} \phi^{j^{\prime} i^{\prime}} & 0
\end{array}\right] \mathbf{V}\right) \\
& -\sum_{i=1}^{N_{\mathrm{s}}} \sum_{j=1}^{N_{\mathrm{r}}} \operatorname{tr}\left(\left[\begin{array}{cc}
0 & \left(g_{1 j}^{*}+(-1)^{i+1} g_{2 j}^{*}\right) \phi^{j i^{H}} \\
0 & 0
\end{array}\right] \mathbf{V}\right) \\
& -\sum_{i=1}^{N_{\mathrm{s}}} \sum_{j=1}^{N_{\mathrm{r}}} \operatorname{tr}\left(\left[\begin{array}{cc}
0 & 0 \\
\left(g_{1 j}+(-1)^{i+1} g_{2 j}\right) \phi^{j i} & 0
\end{array}\right] \mathbf{V}\right) \\
& =\operatorname{tr}(\mathbf{A V}) \text {, }
\end{aligned}
$$

where $\mathbf{A}$ is a matrix representing the summation of all the matrices inside the trace function in (14). So, we have the following optimization problem

$$
\begin{array}{ll}
\underset{\mathbf{V} \succeq 0}{\operatorname{minimize}} & \operatorname{tr}(\mathbf{A V}) \\
\text { subject to } & \mathbf{V}(k, k)=1, k \in\{1,2, \ldots, M+1\} \\
& \operatorname{rank}(\mathbf{V}) \leq 1
\end{array}
$$

The constraint $\operatorname{rank}(\mathbf{V}) \leq 1$ is non-convex and equivalent to

$$
\operatorname{tr}(\mathbf{V})-\beta_{1}(\mathbf{V})=0,
$$
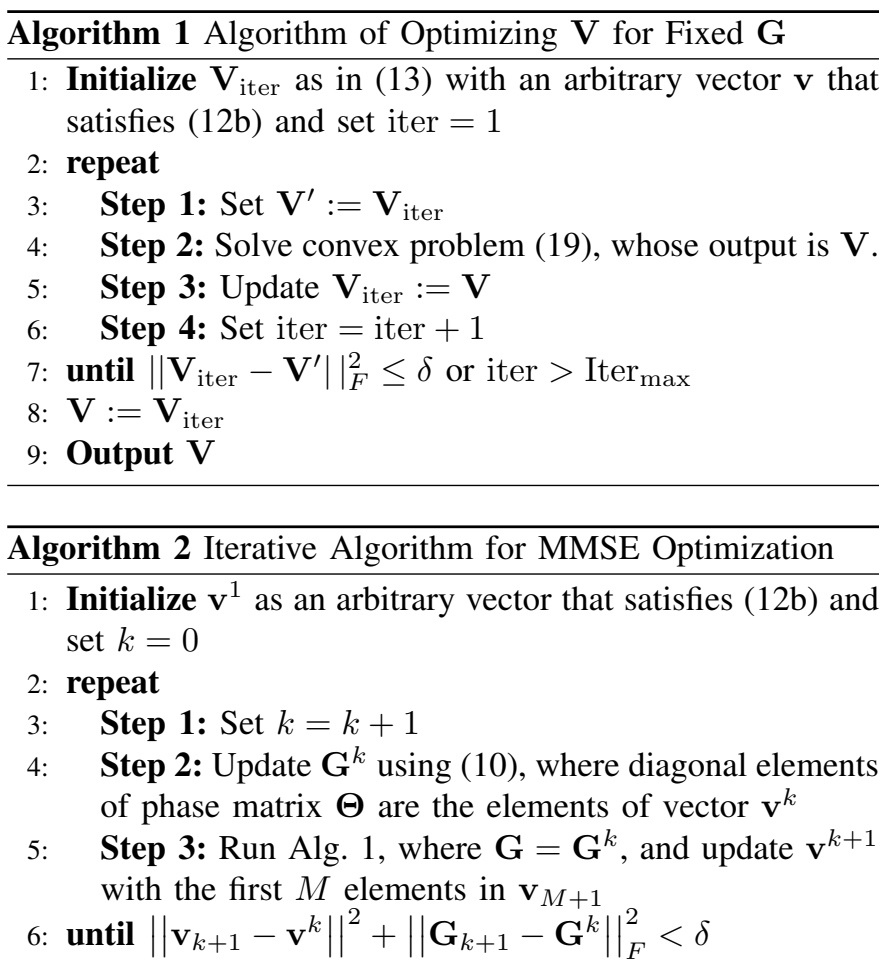

where $\beta_{1}(\mathbf{V})$ is the largest singular value of the matrix $\mathbf{V}$. Also, for $\mathbf{V} \succeq 0$, i.e., semidefinite, the left side of (16) is 0 when $\operatorname{rank}(\mathbf{V}) \leq 1$ is satisfied; otherwise, it is greater than zero. The optimization problem can be written as

$$
\begin{array}{ll}
\underset{\mathbf{V} \succeq 0}{\operatorname{minimize}} & \left(\operatorname{tr}(\mathbf{A V})+\gamma\left(\operatorname{tr}(\mathbf{V})-\beta_{1}(\mathbf{V})\right)\right) \\
\text { subject to } & \mathbf{V}(k, k)=1, k \in\{1,2, \ldots, M+1\},
\end{array}
$$

where $\gamma \geq 0$ is a fixed weight. Since $\beta_{1}(\mathbf{V})$ and $\operatorname{tr}(\mathbf{A V})+$ $\gamma \operatorname{tr}(\mathbf{V})$ are convex functions in $\mathbf{V}$, (17) can be represented as a DC problem. Concave convex procedure (CCP) can efficiently obtain a local optimal solution for DC problems [19]. In this regard, we linearize $-\gamma \beta_{1}(\mathbf{V})$, which induces non-convexity in (17), around a point $\mathbf{V}=\mathbf{V}^{\prime}$ using the following upper bound [12]

$$
-\gamma \beta_{1}(\mathbf{V}) \leq-\gamma \operatorname{tr}\left(\mathbf{V} \mathbf{u}_{\mathbf{1}}\left(\mathbf{V}^{\prime}\right) \mathbf{u}_{\mathbf{1}}\left(\mathbf{V}^{\prime}\right)^{H}\right),
$$

where $\mathbf{u}_{\mathbf{1}}\left(\mathbf{V}^{\prime}\right)$ is the eigenvector of the matrix $\mathbf{V}^{\prime}$ corresponding to its largest eigenvalue, and how to set $\mathbf{V}^{\prime}$ is given in Alg. 1. The equality condition in (18) is satisfied when $\mathbf{V}=\mathbf{V}^{\prime}$. We get the following convex optimization problem, which can be solved by CVx:

$$
\begin{array}{ll}
\min _{\mathbf{V} \succeq 0} & \operatorname{tr}(\mathbf{A V})+\gamma\left(\operatorname{tr}(\mathbf{V})-\operatorname{tr}\left(\mathbf{V} \mathbf{u}_{\mathbf{1}}\left(\mathbf{V}^{\prime}\right) \mathbf{u}_{\mathbf{1}}\left(\mathbf{V}^{\prime}\right)^{H}\right)\right) \\
\text { s.t. } & \mathbf{V}(k, k)=1, k \in\{1,2, \ldots, M+1\} .
\end{array}
$$

Appropriate choice of the penalty parameter $\gamma$ in (19) can be found via simple bisection and remains static throughout Alg. 1. After obtaining the optimal $\mathbf{V}$, vector $\mathbf{v}$ is the first $M$ elements in the last column of $\mathbf{V}$, i.e., $\mathbf{v}_{M+1}$. An iterative algorithm that alternately optimizes the phases of IRS and beamforming matrix $\mathbf{G}$ is given in Alg. 2 . 


\section{OPtimal Detector AND ERROR PERFORMANCE}

The optimal detector and error performance analysis below are derived assuming that the MMSE estimator derived in (10) is the optimal one for the PNC scheme.

\section{A. Optimal Detector}

The likelihood detector to estimate $x_{\oplus}$ from $\mathbf{y}$ can be written, ignoring the noise dependencies in $y_{1}$ and $y_{2}$, as

$$
\begin{aligned}
& \mathcal{L}\left(x_{\oplus} \mid y_{1} y_{2}\right)=\log \left(\frac{\mathbb{P}\left\{y_{1} y_{2} \mid x_{\oplus}=1\right\}}{\mathbb{P}\left\{y_{1} y_{2} \mid x_{\oplus}=-1\right\}}\right) \\
& =\log \left(\frac{\mathbb{P}\left\{y_{1} \mid \widetilde{x}_{1}=0\right\}\left[\mathbb{P}\left\{y_{2} \mid \widetilde{x}_{2}=2\right\}+\mathbb{P}\left\{y_{2} \mid \widetilde{x}_{2}=-2\right\}\right]}{\left[\mathbb{P}\left\{y_{1} \mid \widetilde{x}_{1}=2\right\}+\mathbb{P}\left\{y_{1} \mid \widetilde{x}_{1}=-2\right\}\right] \mathbb{P}\left\{y_{2} \mid \widetilde{x}_{2}=0\right\}}\right) \\
& =2\left(\frac{1}{\sigma_{1}^{2}}-\frac{1}{\sigma_{2}^{2}}\right)+\log \left(\frac{\cosh \left(2 y_{2} / \sigma_{2}^{2}\right)}{\cosh \left(2 y_{1} / \sigma_{1}^{2}\right)}\right),
\end{aligned}
$$

where $\sigma_{i}^{2} \triangleq\left\{\mathbf{G G}^{H}\right\}_{i, i} \sigma^{2}$ is the noise variance on the $i$ th stream after the linear operator. The corresponding decision rule is then

$$
\widehat{x}_{\oplus, \mathrm{r}}=\left\{\begin{array}{ll}
1 & \text { when } \mathcal{L}\left(x_{\oplus} \mid y_{1} y_{2}\right) \geq 0 \\
-1 & \text { when } \mathcal{L}\left(x_{\oplus} \mid y_{1} y_{2}\right)<0
\end{array} .\right.
$$

After that, the relay broadcasts $\widehat{x}_{\oplus, \mathrm{r}}$ to the destinations. At $\mathrm{D}_{1}$, the estimated value of $\widehat{x}_{\oplus \mathrm{r}, \mathrm{r}}$, i.e. $\widehat{x}_{\oplus, \mathrm{D}_{1}}$, is obtained from $y_{\mathrm{D}_{1}}^{r}$ using similar decision rule as in (21) with the likelihood detector $\mathcal{L}_{\mathrm{D}_{1}}^{r}\left(\widehat{x}_{\oplus, \mathrm{r}} \mid y_{\mathrm{D}_{1}}^{r}\right)=2 y_{\mathrm{D}_{1}}^{r} / \sigma_{r, \mathrm{D}_{1}}^{2}$. So, $\mathrm{D}_{1}$ receives $\widehat{x}_{\oplus, \mathrm{D}_{1}}$ and $\widehat{x}_{1}$, which is estimated similarly to $\widehat{x}_{\oplus, \mathrm{D}_{1}}$ with likelihood detector $\mathcal{L}_{\mathrm{D}_{1}}^{\mathrm{S}_{1}}=2 y_{\mathrm{D}_{1}}^{\mathrm{S}_{1}} / \sigma_{\mathrm{S}_{1}, \mathrm{D}_{1}}^{2}$. Then, $\widehat{x}_{2}$ is obtained at $\mathrm{D}_{1}$ by XORing $\widehat{x}_{\oplus, \mathrm{D}_{1}}$ and $\widehat{x}_{1}$. Similarly, $\mathrm{D}_{2}$ receives $\widehat{x}_{2}$ and $\widehat{x}_{\oplus, \mathrm{D}_{2}}$, and obtains $\widehat{x}_{1}$ by XORing the two values.

\section{B. BER Analysis}

Before calculating instantaneous theoretical $\mathbb{P}_{\mathrm{D}_{1}}$ in (7), we can first find the theoretical expressions for instantaneous $\mathbb{P}_{\oplus}^{\mathrm{r}}$, $\mathbb{P}_{\mathrm{D}_{1}}^{\mathrm{S}_{1}}$ and $\mathbb{P}_{\mathrm{D}_{1}}^{\mathrm{r}}$. Let us define the set $\mathbb{X} \triangleq\{-2,2\}$, which contains all the values of $\widetilde{x}_{2}$ and $\widetilde{x}_{1}$ such that $x_{\oplus}=1$ and $x_{\oplus}=-1$, respectively. To derive $\mathbb{P}_{\oplus}^{\mathrm{r}}$ at the relay, we need to further rewrite (20) as

$$
\mathcal{L}\left(x_{\oplus} \mid y_{1} y_{2}\right)=\sum_{i=1}^{N_{\mathrm{s}}}(-1)^{i}\left[\log \left(\sum_{\widetilde{x}_{i} \in \mathbb{X}} e^{-\frac{\left(y_{i}-\widetilde{x}_{i}\right)^{2}}{2 \sigma_{i}^{2}}}\right)+\frac{y_{i}^{2}}{2 \sigma_{i}^{2}}\right]
$$

Now using the soft minimum approximation $\log \left(\sum_{j} \exp \left(-Z_{j}\right)\right) \approx-\min _{j}\left(Z_{j}\right)$ from [20], we can approximate the log likelihood ratio (LLR) in (22) as

$$
\begin{aligned}
\overline{\mathcal{L}}\left(x_{\oplus} \mid y_{1} y_{2}\right) & =\sum_{i=1}^{N_{\mathrm{s}}}(-1)^{i}\left[-\min _{\widetilde{x}_{i} \in \mathbb{X}}\left(\frac{\left(y_{i}-\widetilde{x}_{i}\right)^{2}}{2 \sigma_{i}^{2}}\right)+\frac{y_{i}^{2}}{2 \sigma_{i}^{2}}\right] \\
& =\sum_{i=1}^{N_{\mathrm{s}}}(-1)^{i+1} \min _{\widetilde{x}_{i} \in \mathbb{X}}\left(\frac{-2 y_{i} \widetilde{x}_{i}+\widetilde{x}_{i}^{2}}{2 \sigma_{i}^{2}}\right) .
\end{aligned}
$$

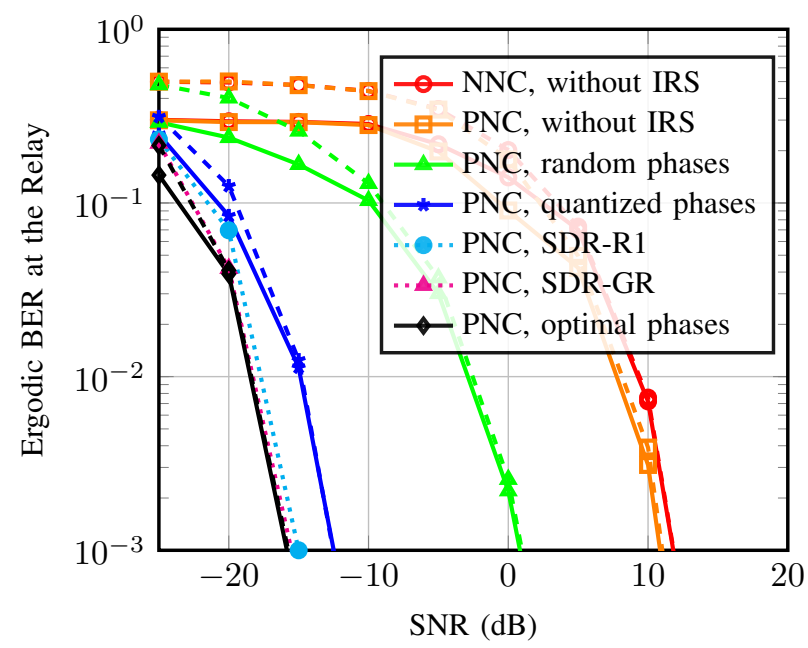

Fig. 2. Ergodic BER at the relay, $\mathbb{P}_{\oplus}^{\mathrm{r}}$, as a function of SNR, for $M=32$.

TABLE I

COMPARISON OF SDR-BASED AND OUR PROPOSED ALGORITHMS

\begin{tabular}{||c|c|c|c||}
\hline Algorithm & MSE & BER at the relay & Relative time \\
\hline \hline Proposed & 0.8201 & 0.0451 & - \\
\hline SDR-R1 & 3.67 & 0.0696 & 0.66 \\
\hline SDR-GR & 0.82015 & 0.0452 & 1.1 \\
\hline
\end{tabular}

Hence, instantaneous $\mathbb{P}_{\oplus}^{\mathrm{r}}$ can be approximated as

$$
\begin{aligned}
\mathbb{P}_{\oplus}^{\mathrm{r}} \approx & \mathbb{P}\left\{x_{\oplus}=1\right\} \mathbb{P}\left\{\overline{\mathcal{L}}<0 \mid x_{\oplus}=1\right\}+\mathbb{P}\left\{x_{\oplus}=-1\right\} \mathbb{P}\left\{\overline{\mathcal{L}} \geq 0 \mid x_{\oplus}=-1\right\} \\
= & \frac{1}{2}\left[Q\left(\frac{-2}{\sigma_{2}}\right)+Q\left(\frac{-2}{\sigma_{1}}\right)\right] Q\left(\frac{\sqrt{1+\frac{\sigma_{2}^{2}}{\sigma_{1}^{2}}}}{\sigma_{2}}\right) \\
& +Q\left(\frac{2}{\sigma_{2}}\right) Q\left(\frac{-3+\frac{\sigma_{2}^{2}}{\sigma_{1}^{2}}}{\sigma_{2} \sqrt{1+\frac{\sigma_{2}^{2}}{\sigma_{1}^{2}}}}\right)+Q\left(\frac{2}{\sigma_{1}}\right) Q\left(\frac{-3+\frac{\sigma_{1}^{2}}{\sigma_{2}^{2}}}{\sigma_{1} \sqrt{1+\frac{\sigma_{1}^{2}}{\sigma_{2}^{2}}}}\right),
\end{aligned}
$$

where $Q(\cdot)$ is the Q-function [21]. Using the channel inversion precoders and likelihood detectors [21], the BERs at $\mathrm{D}_{1}$ for a given channel realization are $\mathbb{P}_{\mathrm{D}_{1}}^{\mathrm{S}_{1}}=Q\left(1 / \sigma_{\mathrm{S}_{1}, \mathrm{D}_{1}}\right)$ and $\mathbb{P}_{\mathrm{D}_{1}}^{\mathrm{r}}=$ $Q\left(1 / \sigma_{r, \mathrm{D}_{1}}\right)$. Now, theoretical $\mathbb{P}_{\mathrm{D}_{1}}$ is expressed in terms of theoretical $\mathbb{P}_{\oplus}^{\mathrm{r}}, \mathbb{P}_{\mathrm{D}_{1}}^{\mathrm{S}_{1}}$ and $\mathbb{P}_{\mathrm{D}_{1}}^{\mathrm{r}}$ as in (7).

\section{Simulation RESUlts}

In this section, we demonstrate the advantages of using IRS and PNC in terms of the BER. The channel coefficients are generated as standard complex Gaussian random variables, and the signal to noise ratio ( $\mathrm{SNR}$ ) is defined as $\mathrm{SNR} \triangleq P / \sigma^{2}$. In all figures, solid and dashed lines represent theoretical, averaged over many realizations of the channels, and Monte Carlo simulation results, respectively. To evaluate the impact of the IRS phase design, the BER at the relay, $\mathbb{P}_{\oplus}^{\mathrm{r}}$, versus SNR is depicted in Fig. 2 for two network coding schemes (PNC and NNC), two architectures (with and without IRS), for various phase designs, i.e., i) optimal: the phases are designed according to the proposed scheme in Section IV; ii) quantized: the optimal phases are quantized to two levels $(0$ or $\pi$ ); iii) random: the phases are selected uniformly at random from 0 to $2 \pi$; iv) SDR-Rl: the phases are optimized using semidefinite relaxation with rank-one approximation; v) $S D R-G R$ : the phases are optimized using semi-definite relaxation with 


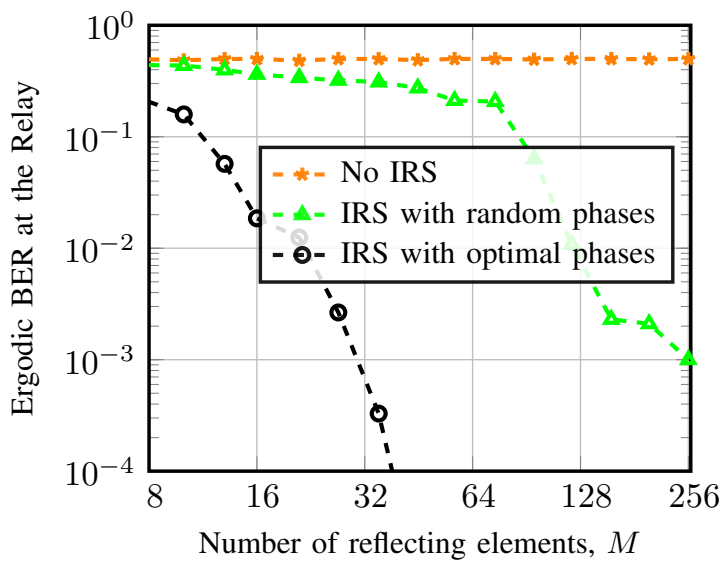

Fig. 3. Ergodic BER at the relay, $\mathbb{P}_{\oplus}^{\mathrm{r}}$, versus the number of reflecting elements at IRS, for $\mathrm{SNR}=-15 \mathrm{~dB}$.

Gaussian randomization [18]. As can be noticed from Fig. 2, implementing just PNC without IRS offers a little SNR gain around $1 \mathrm{~dB}$ over traditional NNC scheme for a target BER of $10^{-3}$. Adding IRS and optimizing its phases can significantly decrease the BER. According to Fig. 2, the PNC approach with optimal phases has SNR gain around $28 \mathrm{~dB}$ over traditional NNC scheme for a target BER of $10^{-3}$. Moreover, the SNR gains compared to quantized phases and random phases are about $3.5 \mathrm{~dB}$ and $17 \mathrm{~dB}$, respectively.

Table I compares our algorithm with SDR-R1 and SDR-GR in terms of MSE and BER at the relay, for $M=32$, SNR= $-20 \mathrm{~dB}, 5000$ Gaussian randomization iterations. Also, the relative computational time (the ratio between computational times of SDR and the proposed algorithm) is shown. Our algorithm and SDR-GR have the best BER performances, but SDR-R1 is the fastest. However, the SDR-GR has higher complexity and slightly slower than the proposed method, as it requires a large number of randomization iterations. The impact of the number of IRS elements on the BER at the relay is shown in Fig. 3. Two phase profiles are considered (optimal and random) along with the case without IRS. For random phases, $\mathbb{P}_{\oplus}^{r}$ decreases slowly, while the optimal phase design allows the BER to decrease with $M$ rapidly.

In Fig. 4, we evaluate BER at $\mathrm{D}_{1}$. We can see that the proposed scheme, i.e., PNC combined with IRS, outperforms the schemes that consider PNC and NNC without IRS. For target $\mathbb{P}_{\mathrm{D}_{1}}=10^{-3}$, the SNR gain of proposed scheme compared to traditional NNC without IRS is around SNR $=0.75 \mathrm{~dB}$. Surprisingly, in contrast to the error experienced at the relay in Fig. 2, the gap between the PNC approaches with different IRS designs is negligible. This is attributed to the fact that $\mathbb{P}_{\mathrm{D}_{1}}^{\mathrm{S}_{1}}$ dominates the effect of $\mathbb{P}_{\oplus}^{\mathrm{r}}$, while computing the BER at $\mathrm{D}_{1}$ in (7). Therefore, future works can consider adding two IRSs to enhance the direct links from the sources to destinations.

\section{CONCLUSION}

We proposed an IRS-aided PNC to improve the BER performance of the wireless network. We derived analytical expressions for the BER. The numerical results show that the proposed scheme significantly enhances the BER performance. As future research directions, our proposed approach can be implemented and analyzed on more complicated network structures with multiple jointly-optimized IRSs.

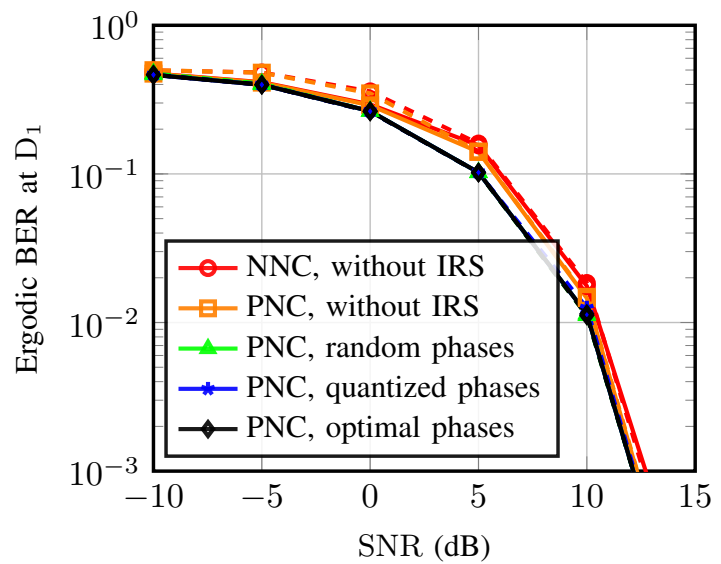

Fig. 4. Ergodic BER at $\mathrm{D}_{1}, \mathbb{P}_{\mathrm{D}_{1}}$, as a function of $\mathrm{SNR}$, for $M=32$.

\section{REFERENCES}

[1] J. Guo, S. Durrani, X. Zhou, and H. Yanikomeroglu, "Massive machine type communication with data aggregation and resource scheduling," IEEE Trans. Commun., vol. 65, pp. 4012-4026, 2017.

[2] S. Katti, H. Rahul, W. Hu, D. Katabi, M. Medard, and J. Crowcroft, "XORs in the air: practical wireless network coding," IEEE/ACM Trans. Netw., vol. 16, pp. 497-510, 2008.

[3] J. Sykora and A. Burr, Wireless Physical Layer Network Coding. Cambridge University Press, 2018.

[4] O. Kosut, L. Tong, and D. Tse, "Nonlinear network coding is necessary to combat general byzantine attacks," in Proc. 47th Annu. Allerton Conf. Commun. Control Comput., 2009, pp. 593-599.

[5] N. Lee, J.-B. Lim, and J. Chun, "Degrees of freedom of the MIMO Y channel: Signal space alignment for network coding," IEEE Trans. Inf. Theory, vol. 56, pp. 3332-3342, 2010.

[6] S. Zhang and S. C. Liew, "Physical layer network coding with multiple antennas," in Proc. IEEE Wireless Commun. Netw. Conf., 2010.

[7] Q. Wu and R. Zhang, "Intelligent reflecting surface enhanced wireless network: Joint active and passive beamforming design," in Proc. IEEE Global Commun. Conf. (GLOBECOM), 2018.

[8] M. Di Renzo et al., "Smart radio environments empowered by reconfigurable AI meta-surfaces: An idea whose time has come," EURASIP J. Wireless Commun. Netw., vol. 2019, p. 129, 2019.

[9] C. Huang et al., "Reconfigurable intelligent surfaces for energy efficiency in wireless communication," IEEE Trans. Wireless Commun., vol. 18, pp. 4157-4170, 2019.

[10] H. Ibraiwish et al., "EMF-aware cellular networks in RIS-assisted environments," 2021. [Online]. Available: http://hdl.handle.net/10754/666963

[11] A. Elzanaty et al., "Towards 6G holographic localization: Enabling technologies and perspectives," arXiv, 2021.

[12] T. Jiang and Y. Shi, "Over-the-air computation via intelligent reflecting surfaces," in Proc. IEEE Global Commun. Conf. (GLOBECOM), 2019

[13] D. Yu et al., "Optimizing over-the-air computation in IRS-aided C-RAN systems," in Proc. IEEE 21st Int. Workshop Signal Process. Advances Wireless Commun. (SPAWC), 2020.

[14] A. Elzanaty et al., "Reconfigurable intelligent surfaces for localization: Position and orientation error bounds," IEEE Trans. Signal Process., 2021, [Early Access].

[15] W. Fang et al., "Stochastic beamforming for reconfigurable intelligent surface aided over-the-air computation," in Proc. IEEE Global Commun. Conf. (GLOBECOM), 2020.

[16] R. Ahlswede, N. Cai, S.-Y. R. Li, and R. W. Yeung, "Network information flow," IEEE Trans. Inf. Theory, vol. 46, pp. 1204-1216, 2000.

[17] M. P. Wilson, K. Narayanan, H. D. Pfister, and A. Sprintson, "Joint physical layer coding and network coding for bidirectional relaying," IEEE Trans. Inf. Theory, vol. 56, pp. 5641-5654, 2010.

[18] Z.-Q. Luo et al., "Semidefinite relaxation of quadratic optimization problems," IEEE Signal Process. Mag., vol. 27, pp. 20-34, 2010.

[19] M. Tao, E. Chen, H. Zhou, and W. Yu, "Content-centric sparse multicast beamforming for cache-enabled cloud RAN," IEEE Trans. Wireless Commun., vol. 15, pp. 6118-6131, 2016.

[20] G. C. Calafiore, S. Gaubert, and C. Possieri, "A universal approximation result for difference of log-sum-exp neural networks," IEEE Trans. Neural Netw. Learning Syst., vol. 31, pp. 5603-5612, 2020.

[21] J. Proakis and M. Salehi, Digital Communications. McGraw-Hill, 2008. 